\title{
HPLC Method for Separation of Cannabidiol Hemp Seed Oil with Skin Lipids and Tandem HRMS Technology for Characterization of a Chemical Marker
}

\author{
Yuxin Liu, Yu Wang, Zhigang Hao * and Long Pan \\ Cross Category Research \& Innovation Department, Technology Center, Colgate-Palmolive Company, \\ 909 River Rd, Piscataway, NJ 08807, USA; y1971@scarletmail.rutgers.edu (Y.L.); ynjwang@ucdavis.edu (Y.W.); \\ long_pan@colpal.com (L.P.) \\ * Correspondence: zhigang_hao@colpal.com
}

check for updates

Citation: Liu, Y.; Wang, Y.; Hao, Z.; Pan, L. HPLC Method for Separation of Cannabidiol Hemp Seed Oil with Skin Lipids and Tandem HRMS Technology for Characterization of a Chemical Marker. Cosmetics 2021, 8 , 108. https://doi.org/10.3390/ cosmetics 8040108

Academic Editors: Amparo Salvador Carreño and Juan Luis Benedé Veiga

Received: 15 October 2021

Accepted: 14 November 2021

Published: 17 November 2021

Publisher's Note: MDPI stays neutral with regard to jurisdictional claims in published maps and institutional affiliations.

Copyright: (c) 2021 by the authors. Licensee MDPI, Basel, Switzerland. This article is an open access article distributed under the terms and conditions of the Creative Commons Attribution (CC BY) license (https:// creativecommons.org/licenses/by/ $4.0 /)$.

Abstract: Cannabidiol (CBD) hemp seed oil is a commercial raw material with antioxidant and anti-inflammatory benefits that has been formulated into body wash and skin care products. The biggest analytical challenge is how to simultaneously quantify CBD and hemp seed oil as they deposited on the skin surface. CBD is easily separated and quantified from skin surface extracts via a HPLC-mass spectrometry methodology. However, the structural skeleton of triacylglycerides (TAGs) in hemp seed oil is same as those from the skin surface sebum. The strong hydrophobicity with subtle structural difference challenges their separation. In this project, a new reverse phase HPLC-high resolution mass spectrometry methodology was developed with a strong mobile phase normal propanol. The separated hemp seed oil TAGs in the chromatogram were identified and characterized using data-dependent acquisition (DDA) technology. Based on the daughter ion characterization, the separated peak with an ammonium adduct at $890.7226\left[\mathrm{M}+\mathrm{NH}_{4}\right]^{+}$was confirmed as the parent ion of glycerol with three omega-3 fatty acid chains. This is the first time TAG structure with direct HPLC-tandem mass spectrometry technology has been elucidated without a hydrolysis reaction. The confirmed TAG structure with an ammonium adduct at $890.7226 \pm 0.0020$ can be used as a representative chemical marker for the hemp seed oil quantification.

Keywords: cannabidiol; hemp seed oil; skin lipids; triacylglycerides; liquid chromatography; high resolution mass spectrometry; data-dependent acquisition

\section{Introduction}

CBD has been comprehensively studied with the conclusion that even high doses of CBD are safe and are well tolerated without causing significant side effects [1,2]. Recently, $\mathrm{CBD}$ as an active ingredient has been investigated in clinical trials for the treatment of many medical conditions. The application of CBD in the cosmetic industry has also grown rapidly [3]. Anti-inflammatory properties enhance the therapeutic effects of acne vulgaris, since it counteracts multiple pathogenic factors of acne: sebum over-production, and sebocyte over proliferation [4]. In addition, its antioxidation function is able to help counteract oxidative cell damage generated by free radicals, decreasing visible signs of skin aging [5]. Those benefits have made CBD become an important ingredient in the cosmetic industry in recent years [6,7]. Both current European legislation and US FDA regulation do not prohibit the use of synthetically obtained CBD in cosmetic products.

Hemp seed oil is well known to contain the rich omega-3 fatty acids and their triacylglycerides (TAGs). Omega-3 is an essential polyunsaturated fatty acid (PUFA) and its main derivatives including triacylglycerides have been widely used as antioxidants and anti-inflammatory agents in the cosmetic industry [8,9]. Clinically, omega-3 based lipids can benefit patients with chronic plaque psoriasis [10]. As commercial raw materials, CBD has been blended into hemp seed oil for the cosmetic product application. 
The challenge to simultaneously quantify CBD and hemp seed oil TAG was reported in our previous study [11]. We found that the common mobile phases like methanol and acetonitrile cannot effectively elute TAGs out from reverse phase columns. The accumulation of TAGs inside the column builds up the column pressure and generates ghost peaks even with the high-resolution mass detector as the filter. Strategically, a hydrophilic interaction chromatography (HILIC) column with an extremely high organic mobile phase was applied to avoid the strong retention of hydrophobic TAG ingredients. However, the method was not able to accurately quantify the hemp seed oil TAG, especially during the skin deposition study. TAG ingredients from skin and hemp seed oil could interfere with each other and result in poor separation. To effectively develop a reliable method to simultaneously quantify both CBD and hemp seed oil, skin lipid chemistry was reviewed. The animal and human skin surface, the first barrier against external environment, is covered by sebum oil. Sebum is secreted by the sweat glands and located on the outermost surface layer of skin. The TAG contents in sebum can reach up to $50 \%$ according to previous studies [12,13]. Other skin lipids such as ceramides, phospholipids and cholesterol are also present on the surface of skin, but our data indicated that no significant amount of those ingredients could be extracted by ethanol directly. Trypsin enzyme treatment is necessary if these lipids need to be extracted. TAG is considered a minor lipid species of lamellar bodies and has received little attention in critical reviews [14] However, recent discoveries demonstrated that dysfunction and deficiencies of enzymes and activators of TAG synthesis and catabolism can cause major skin defects in mice and humans. The findings summarized in the review suggested that the crucial role of neutral lipid metabolism in the generation of a functional permeability barrier in skin.

Historically, huge separation efforts have been given to TAG from traditional normal phase to reversed phase HPLC separation $[15,16]$, plus argentation (silver ion) and two dimensional $(\mathrm{LC} \times \mathrm{LC})$ methods $[17,18]$. High temperature gas chromatography (HTGC) has often been used $[19,20]$. Due to large variation of fatty acid chain lengths, saturation levels and steric isomers, 64 million TAG structures can be present in nature [21]. Therefore, no single method can be used for all applications. In this project, our goals were to differentiate the TAGs from CBD hemp seed oil from the skin surface and establish a reliable chemical marker for quantification. A normal phase HPLC-MS method was tested first. Unfortunately, organic mobile phases such as hexane and ethyl acetate were unhelpful and derailed our efforts. To improve mobile phase elution capability, a reverse phase column combined with normal propanol was applied. At same time, a glyceryl trinonadecanoate standard was used to monitor the elution power and make sure the main natural TAG ingredients were not accumulated inside the column. Finally, one chemical marker for hemp seed oil quantification was successfully separated from all TAG ingredients presented in pig skins. The next challenge was how to characterize the separated TAG peak as a chemical marker for CBD hemp seed oil quantification. The TAG structures can be profiled and characterized by using chromatographic techniques [11,21]. Fragmentation patterns and neutral loss in mass spectrometry have been used for the quantification of regioisomers with minimal standards [22,23], and the online acetone Paterno-Buchi reaction and ultraviolet photo-dissociation coupled with mass spectrometry was recently used to determine the double bond position in TAG structures [24,25]. Detailed chemical marker structure in the current project was identified and characterized by tandem mass spectrometry combined with fragmentation patterns and literature profiles. The developed method can be used to quantify both CBD and hemp seed oil during the skin deposition in the future.

\section{Materials and Methods}

\subsection{Apparatus}

High-pressure liquid chromatography-heated electrospray ionization-high resolution mass spectrometry (HPLC-HESI-HRMS) analysis was performed using a Q-Exactive ${ }^{\mathrm{TM}}$ Orbitrap $^{\mathrm{TM}}$ mass spectrometry equipped with a HESI-II interface and Vanquish HPLC Systems from Thermo Fisher Scientific (San Jose, CA, USA). An Eppendorf centrifuge 
5810R was used to centrifuge the skin sample for $10 \mathrm{~min}$ at $500 \times \mathrm{g}$ before HPLC-HRMS analysis. A Genie 2 vortex mixer was used to assist with sample dispersion, minimizing preparation time.

\subsection{Reagents and Samples}

HPLC-grade normal propanol, methanol and ethanol were purchased from SigmaAldrich (St. Louis, MO, USA), and chloroform was purchased from J.T. Baker (Center Valley, PA, USA). Water was purified by using an ELGA PURELAB Prima ultrapure water purification system from MilliPoreSigma (Burlington, MA, USA). A 1× Trypsin-EDTA solution from Sigma-Aldrich containing $0.5 \mathrm{~g}$ porcine trypsin and $0.2 \mathrm{~g}$ EDTA-4Na per liter of Hanks' Balanced Salt Solution with phenol red, was 1:1 diluted with water before use. Cannabis sativa L. CBD hemp seed oil (5\%) was purchased from International Cosmetics Science Center (Harlev J, Demark) [26]. Pig skin was a product from an abattoir supplied by Animal Technologies (Tyler, TX, USA).

\subsection{Chemical Standard Preparation}

An amount of $285 \mathrm{mg}$ of 5\% CBD-blended hemp seed oil reference standard was weighed into a $100 \mathrm{~mL}$ volumetric flask and dissolved in chloroform as a stock solution. An amount of $270 \mathrm{mg}$ glyceryl trinonadecanoate from Sigma-Aldrich (purity $\geq 99 \%$ ) was weighed into a $100 \mathrm{~mL}$ volumetric flask and dissolved in chloroform as a stock solution. The stock solutions were stored at $-20{ }^{\circ} \mathrm{C}$ for a week until use. The detailed dilution procedures from stock solution to working solution are shown in Table 1. Diluted standard working solutions were transferred into $1.8 \mathrm{~mL}$ HPLC vials for HPLC-MS analysis. Due to the strong hydrophobicity of TAG standards, chloroform was used in stock solution. To reduce potential diluent impact on initial mobile phase, 50\% normal propanol in water was used at the end of dilution.

Table 1. Preparation of CBD-blended hemp seed oil (HSO) reference and glyceryl trinonadecanoate (GTND) chemical standards.

\begin{tabular}{ccccc}
\hline CBD Hemp Seed Oil & Dilution Ratio & Diluent & HSO Concentration & CBD Concentration \\
\hline Stock solution (S0) & & Chloroform solvent & $2850 \mathrm{ppm}$ & $142,500 \mathrm{ppb}$ \\
\hline Working solution S1 & $1: 100$ from S0 & $1: 10$ chloroform:normal propanol solvent & $28.5 \mathrm{ppm}$ & $1425 \mathrm{ppb}$ \\
\hline Working solution S2 & $1: 10$ from S1 & $80 \%$ normal propanol water solvent & $5.7 \mathrm{ppm}$ & $285 \mathrm{ppb}$ \\
\hline Working solution S3 & $1: 4$ from S2 & $50 \%$ normal propanol water solvent & $0.425 \mathrm{ppm}$ & $71.3 \mathrm{ppb}$ \\
\hline Working solution S4 & $1: 4$ from S3 & $50 \%$ normal propanol water solvent & $0.356 \mathrm{ppm}$ & $17.8 \mathrm{ppb}$ \\
\hline Working solution S5 & $1: 4$ from S4 & $50 \%$ normal propanol water solvent & $0.089 \mathrm{ppm}$ & Concentration \\
\hline GTND Standard & Dilution Ratio & Diluent & $2700 \mathrm{ppm}$ \\
\hline Stock solution (S0) & & chloroform & $135 \mathrm{ppm}$ \\
\hline S1 & $1: 20$ from S0 & $1: 10$ chloroform:normal propanol solvent & $1.35 \mathrm{ppm}$ \\
\hline S2 & $1: 100$ from S1 & $1: 10$ chloroform:normal propanol solvent & normal propanol & $0.135 \mathrm{ppm}$ \\
\hline S3 & $1: 10$ from S2 & $1: 5$ from S3 & $50 \%$ normal propanol water solvent & $0.027 \mathrm{ppm}$
\end{tabular}

\subsection{Skin Sample Preparation}

Pig skin was cut into $5 \mathrm{~cm} \times 5 \mathrm{~cm}$ square pieces and stored at $-80{ }^{\circ} \mathrm{C}$ until use. To extract the pig skin surface lipids, skin tissues were taken from freezer and kept at room temperature for one hour. A hollow round-bottom glass cup was put on the skin surface as shown in Figure 1. One milliliter of ethanol was pipetted into the glass cup and stirred with a glass rod for $1 \mathrm{~min}$. The extract was transferred with a pipette and this step was repeated for a total of three extractions (pooled) per piece. This procedure was repeated with one additional piece. Finally, all extracted samples were combined and dried under nitrogen in a manifold. The dried residues were redissolved in $2 \mathrm{~mL}$ of chloroform: normal propanol 
at a 1:4 ratio and diluted with 50\% normal propanol-water solution at 1:100 ratio. The solution was filtered through a $0.2 \mu \mathrm{m}$ PTFE membrane before analysis by HPLC-HRMS. The glass cup was cleaned with ethanol and sonication after lipid extraction. The extraction protocol was modified from previous studies $[27,28]$.

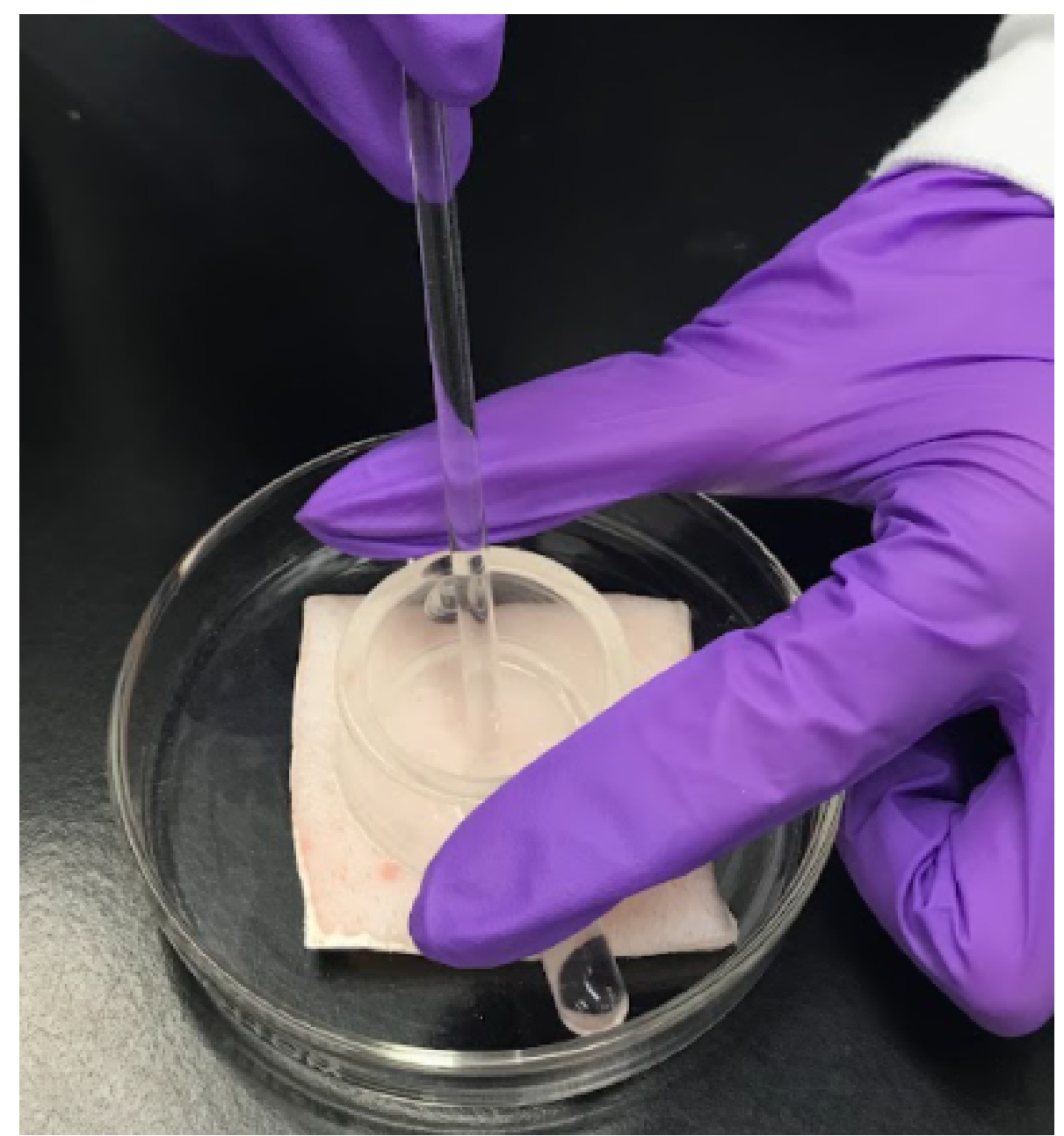

Figure 1. Pig skin surface lipid extraction via a hollow glass cup using ethanol as the extraction solvent.

To confirm the location of lipids other than TAG, and to understand their impact on hemp seed oil deposition, an enzyme treatment was conducted for lipid extraction. Specifically, one piece of the skin was put into a petri dish with the stratum corneum sidedown, $5 \mathrm{~mL}$ of a 1 to 1 diluted trypsin buffer was added, followed by incubation at $37{ }^{\circ} \mathrm{C}$ overnight. The enzyme treated pig skin pieces were then saved for extraction. The solution was removed from the petri dish and transferred into a $15 \mathrm{~mL}$ falcon tube, followed by centrifugation at $500 \times g$ for $5 \mathrm{~min}$. The supernatant was discarded, and the cell pellet was resuspended and washed with water, centrifugation was repeated, and the cell pellet was stored for extraction. Lipids extractions from collected cell pellets and the trypsin treated pig skin were performed based on a modified method reported previously by Wertz and Downing [29]. In short, three mixtures of chloroform: methanol (2:1, 1:1, 1:2) were added to the cell and skin sheets separately for $90 \mathrm{~min}$ each, followed by overnight extraction with methanol. Notably, for skin sheets extractions, phase separation was observed due to water content in the tissue. The upper phase containing polar molecules (salts, amino acids) was discarded, as per the Bligh and Dyer extraction methodology. Extracts from cells and skin sheets were combined and filtered for analysis by HPLC-HRMS. 


\subsection{High Performance Liquid Chromatography-Mass Spectrometry Analysis}

Three microliters of sample or standard solutions were injected into a Waters XBridge $\mathrm{C}_{18}(2.1 \times 150 \mathrm{~mm}, 3.5 \mu \mathrm{m})$ column. Mobile phase A was pure water while mobile phase B was normal propanol. The method was set at $50 \% \mathrm{~B}$ from 0 to $1 \mathrm{~min}$ and a gradient from $50 \%$ to $99 \%$ B from 1 to $36 \mathrm{~min}$ and kept at $99 \%$ B until $42 \mathrm{~min}$. The column was equilibrated to $50 \%$ at $41 \mathrm{~min}$ and held isocratic until $50 \mathrm{~min}$. The total run time was $50 \mathrm{~min}$ with a flow rate of $0.2 \mathrm{~mL} / \mathrm{min}$. Samples were analyzed in full scan MS mode from 133.4 to $2000 \mathrm{~m} / \mathrm{z}$ under positive polarity with electrospray ionization for CBD and TAGs. The resolution of 70,000 FWHM with $2.0 \times 10^{6}$ of Automatic Gain Control (AGC) target and $100 \mathrm{~ms}$ of maximum ion injection time was fixed during the analysis. Analyses were performed without a lock mass and protonated molecular weights at 315.2319 Daltons and quaternary ammonium TAG molecular weights at 890.7226 Daltons were used to quantify CBD and hemp seed oil. The optimized parameter settings were sheath, auxiliary and curtain gas flow rates at 35, 10 and 8 arbitrary units, respectively, a spray voltage of $3.75 \mathrm{kV}$, a capillary temperature of $320^{\circ} \mathrm{C}$, an S-lens RF level 50, and an auxiliary gas heater temperature $400{ }^{\circ} \mathrm{C}$. Software used for operating the HILIC-HRMS was Xcalibur ${ }^{\mathrm{TM}}$ (version 4.1).

For data-dependent acquisition (DDA), the mass spectrometer was operated in positive ion mode. The survey scans were acquired at a resolution of 70,000 with a maximum injection time of $100 \mathrm{~ms}$ and a target value of $3 \times 10^{6}$ over a mass range of $67 \sim 1000 \mathrm{~m} / \mathrm{z}$. The target candidate masses were selected from the separated TAG into inclusion lists $(890.7226,892.7375,894.7525$ and 896.7682). The quaternary ammonium adducts, and their corresponding sodium adducts $(895.6727,897.6890,899.7042$ and 901.7198) are listed in an exclusion table. The fragments were recorded at a resolution of 17,500 with an injection time of $50 \mathrm{~ms}$ and a target value of $1 \times 10^{5}$. The isolation window was set at $4 \mathrm{~m} / \mathrm{z}$ and the NCE was at 30 .

\section{Results and Discussion}

\subsection{Chemical Standard Preparation}

Glycerol trinonadecanoate, as a mobile phase elution capability indicator, is a very hydrophobic TAG compound. Its solubility in propanol and water mixtures is limited. To follow homogenization rules during sampling procedures, Glycerol trinonadecanoate TAG stock solution $\mathrm{S} 0$ was made with a chloroform solvent to make sure it could be completely solubilized. Chloroform has very strong elution power in reverse phase chromatography. To avoid the distortion of mobile phase elution power during the injection, the diluent polarity was adjusted to be close to the initial mobile phase composition ( $50 \%$ normal propanol in water) at the end. To balance this TAG solubility and compatibility to initial mobile phase composition, gradient diluents were applied. The detailed dilution procedure is shown in Table 1. A similar sampling procedure was conducted for hemp seed oil; its details are described in Table 1.

\subsection{Skin Sample Preparation}

The hemp seed oil deposition amount was usually limited after sample treatment and washout. Therefore, a more environmentally friendly solvent, ethanol, was selected to extract the pig skin lipid. The extract analyses from pig skin surfaces with ethanol indicated that major skin lipids are composed of TAG from sebum oil, as described in a previous study [13]. No significant amounts of other types of skin lipids, such as phospholipids, cholesterol and ceramides, were observed.

To better understand the absence of those types of skin lipids with ethanol extraction, the pig skin was treated with trypsin enzyme. This enzyme can effectively dissociate the physicochemical interaction of skin lipids from the skin matrices. After enzyme treatment, a significant amount of fatty acids, phospholipids, cholesterol and ceramides could be extracted by ethanol. Detailed results are discussed in Section 3.4. 


\subsection{Liquid Chromatographic Conditions}

The skin surface is the outermost protective barrier of our body. The skin surface is coated with a lipid film composed of a mixture of sebaceous lipids (the sebum oil) and a small fraction of epidermal lipids that can be detached from the outermost layer of the skin, stratum corneum. Those extracted skin lipids can interfere with analytical results. The saturation level of the TAG fatty acid chain in pig and human skins generally is higher than TAG from botanical sources. Due to the high hydrophobicity of TAG ingredients present in hemp seed oil and the skin surface, strong elution power mobile phases such as hexane and chloroform should be chosen $[15,16]$ but their high volatility can reduce method reproducibility. More practically, our Thermo Vanquish HPLC System cannot handle such aggressive organic solvents. Therefore, a safer and more environmentally friendly solvent, normal propanol, was applied. To simultaneously quantify CBD and hemp seed oil for the skin deposition study, a gradient from 50\% to $99 \%$ normal propanol was considered. Extra elution time with $99 \%$ normal propanol was afforded to elute all TAG ingredients out of the column. A TAG standard with three $C_{19}$ saturated fatty acid chains, glycerol trinonadecanoate, at a slightly higher concentration was used as an indicator for method development, as shown in Figure 2A, to ensure most TAG ingredients with $C_{14}$ to $C_{18}$ fatty acid chains from hemp seed oil and skin surface could be eluted from the column. CBD can be easily differentiated from skin surface ingredients based on polarity considerations. Retention of CBD is $5.27 \mathrm{~min}$ of the gradient period, and most representative TAG ingredients have retention times less than $40 \mathrm{~min}$. It should be noted that TAG ingredients from hemp seed oil and skin surface are very similar because they have the same structural skeleton with one glycerol plus three fatty acid chains. The double bond variation is a relatively weak factor for TAG separation. The results shown in Figure 2B,C indicate that normal propanol is a very effective mobile phase solvent to separate those TAGs. The peaks from 32.40 to 36.40 min were present in both hemp seed oil and skin lipids. However, the peaks at retention times of 30.32 and 31.08 min were only present in hemp seed oil, and can be considered as chemical markers for hemp seed oil during the skin deposition study.

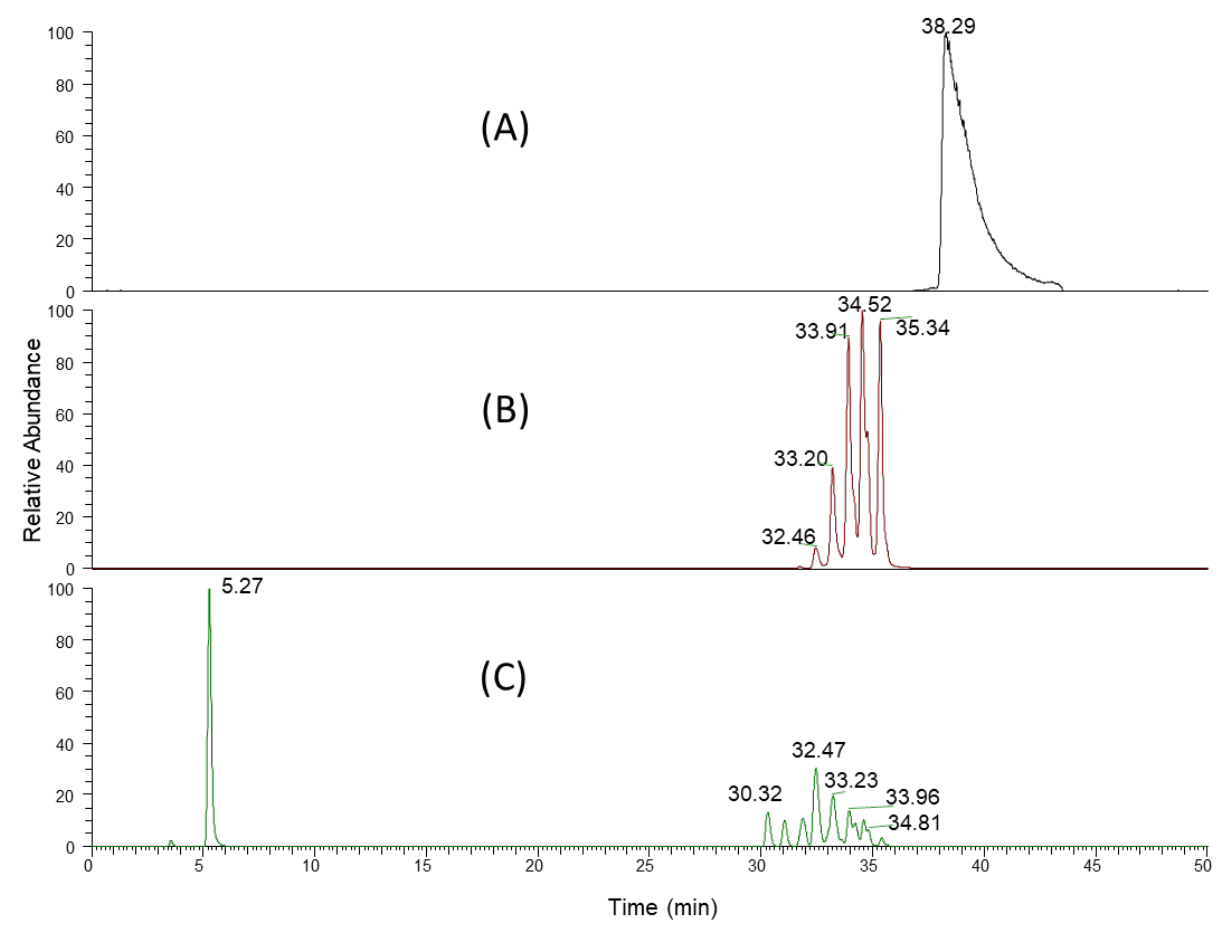

Figure 2. Representative chromatograms obtained by applying the proposed method. (A) Glycerol trinonadecanoate standard; (B) pig skin extracts; (C) CBD-blended hemp seed oil reference standard. 
For the trypsin enzyme-treated pig skin surface materials, more lipid ingredients were extracted by ethanol. A typical chromatogram of ceramides with $\mathrm{C}_{2} \mathrm{H}_{4}$ variation is shown in Figure 3. The natural fatty acid chains are dominant in even-numbered carbons, and they differ from each other with a $\mathrm{C}_{2} \mathrm{H}_{4}$ unit due to their biosynthetic pathway. The exact carbon chain lengths in ceramides structures were assigned by accurate molecular weight and have not been confirmed by MS fragmentation. Those peaks were not observed in direct ethanol extracts either from pig or human skin surfaces, which could be related to the location difference of various skin lipids and their physiochemical interactions.

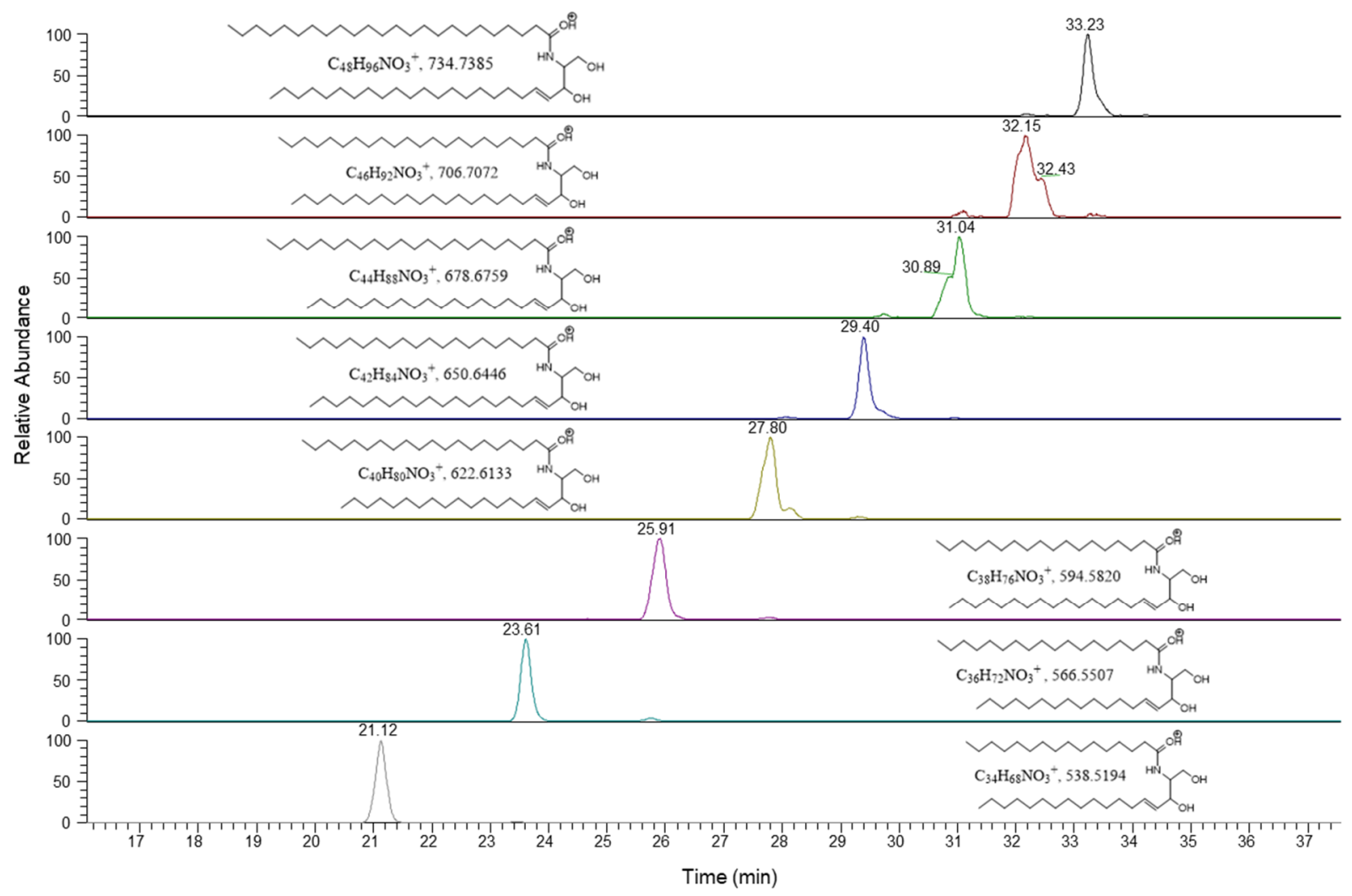

Figure 3. HRMS chromatograms of ceramides in pig skin after trypsin enzyme treatment. Two fatty chain lengths could be varied.

\subsection{High Resolution Mass Spectra}

A reversed phase $\mathrm{C}_{18}$ column combined with a strong gradient mobile phase provided good separation for both CBD and sequential TAG ingredients, as shown in Figure 2. The corresponding high resolution mass spectra (Figure 4) exhibited a variable molecular weights and double bond numbers of TAG ingredients for the peak retention times ranging from 30.32 to $35.43 \mathrm{~min}$. The even numbers for each peak mass signal, such as 890 and 892 predicted nitrogen-containing molecular adducts based on the nitrogen rule in mass spectrometry. In addition, the odd MS numbers on the right of those even numbers, such as 895 and 897 shown in Figure 4, are corresponding sodium adducts $[\mathrm{M}+\mathrm{Na}]^{+}$. The highresolution mass data can help to determine the chemical formula of each peak. However, the detailed structures could not be assigned at this stage due to the alternative chain lengths and double bond positions in TAG compounds. 


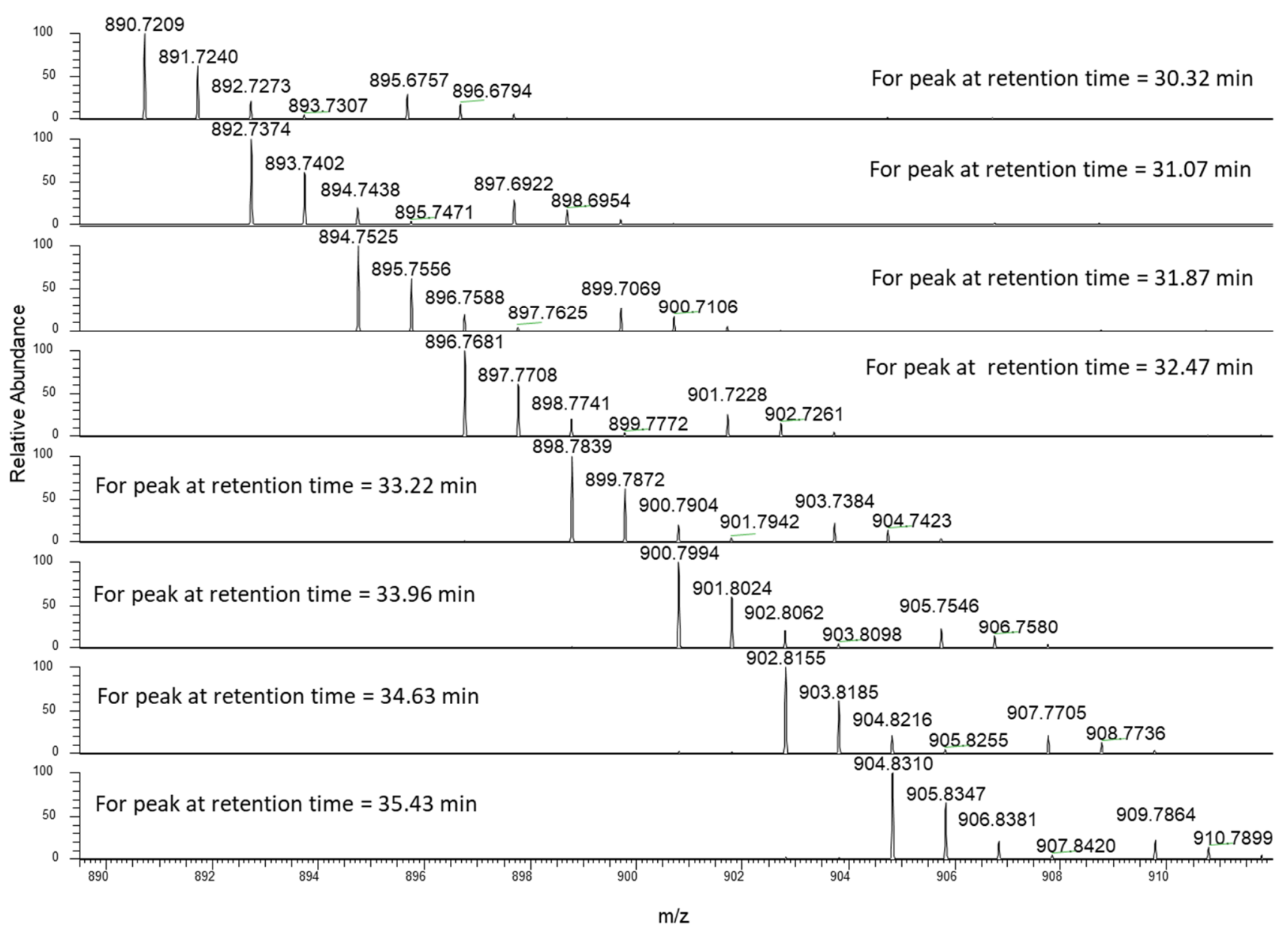

Figure 4. TAG molecular ammonium adduct $\left[\mathrm{M}+\mathrm{NH}_{4}\right]^{+}$corresponding to the peaks in Figure 2.

\subsection{Structural Identification of Chemical Marker for Hemp Seed Oil}

Linolenic and linoleic acids are two essential fatty acids that cannot be synthesized by humans and other animals [30]. TAGs composed of linolenic and linoleic fatty acid chains are dominant ingredients in hemp seed oil, which can be distinguished from the TAG ingredients from the pig skin surface. Those ingredients are critical for hemp seed oil benefits such as antioxidant and anti-inflammatory functions for healthy skin. At the same time, they can be chemical markers for the hemp seed oil quantification with the skin surface matrix. In this study, by using a water-normal propanol gradient elution in a $\mathrm{C}_{18}$ column, two TAG peaks with retention times of 30.32 and 31.08 min were completely separated from other TAG peaks at 32 to $37 \mathrm{~min}$ as seen in Figure 2. To confirm their TAG fatty acid chain composition, a tandem mass spectrometry, data-dependent acquisition (DDA) was run for TAG ammonium adducts (Figure 4) to observe their daughter ion, as shown in Figure 5. 


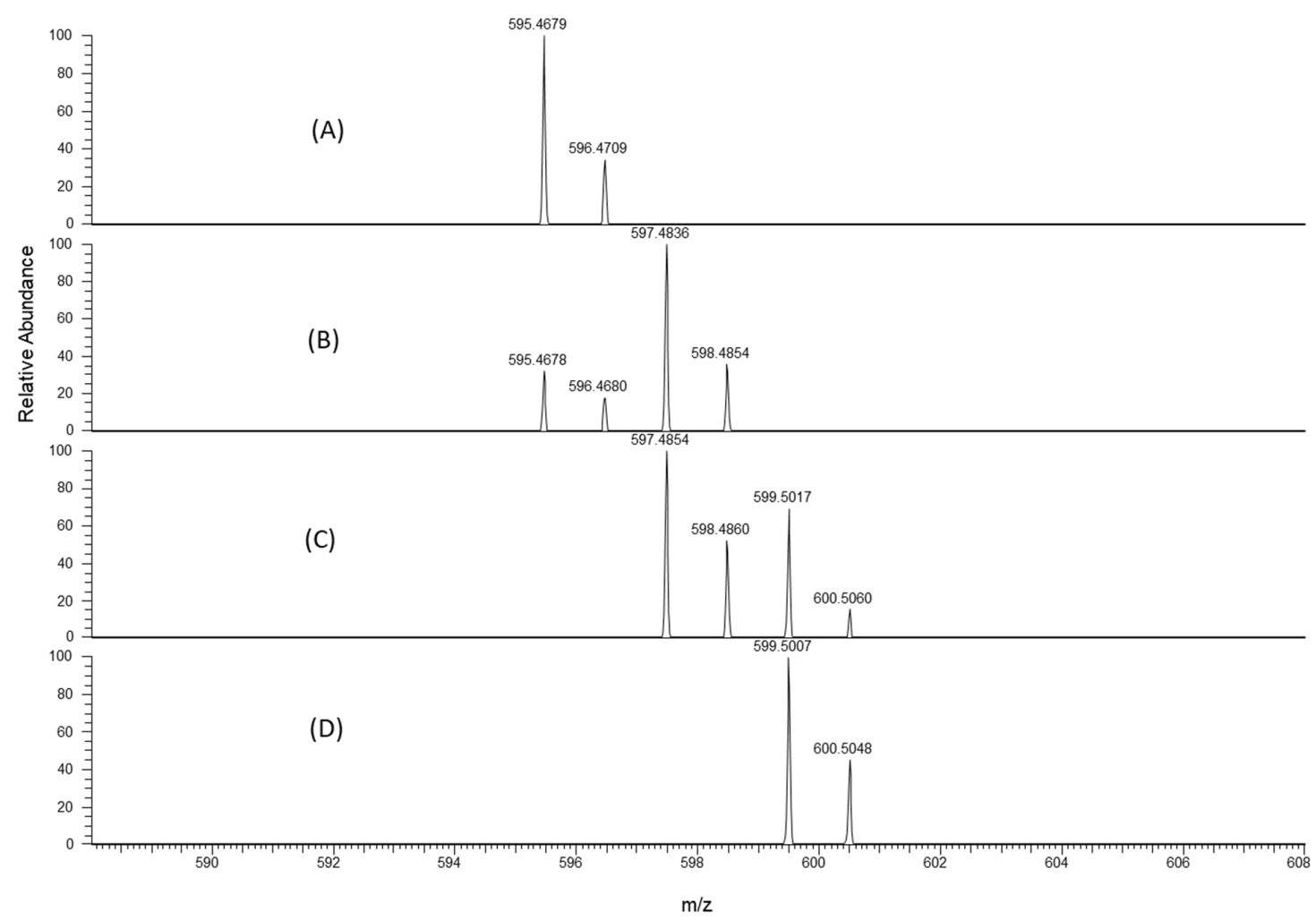

Figure 5. Daughter ions from their corresponding parent ions of TAG ingredients shown in Figure 4. (A) The fragments from 890.7216 parent ions; (B) The fragments from 892.7375 parent ions; (C) The fragments from 894.7525 parent ions; (D) The fragments from 896.7682 parent ions.

For the parent ion of 890.7216 shown in Figure 4, corresponding to the peak at $30.32 \mathrm{~min}$ in Figure 3, only one daughter ion was observed at 595.4679 Daltons with 596.4709 Dalton peak being the isotopic match, as shown in Figure 5, when normalized collision energy (NCE) at 30 was applied. Its TAG structure and fragmentation pathways are shown in Figure 6. If all fatty acid chains have the same weight, its fragments A, B and $C$ should have the same composition. The TAG structure should have three similar linolenic fatty acid chains linked to glycerol, based on the hemp seed oil fatty acid profile reported in the literature [31-34]. However, if the red color linolenic fatty acid chain with three double bonds is changed into a linoleic chain with the two double bonds, fragments $A$ and B should have a weight of 597.4836 Daltons and fragment $C$ should keep its weight at 595.4679 Daltons. Their intensity ratio should be 2:1, which was confirmed by the peak intensity as shown in Figure 5B. Therefore, the TAG peak at 31.07 min with the ammonium adduct of 892.7375 Daltons should contain two linolenic fatty acid chains and one linoleic fatty acid chain.

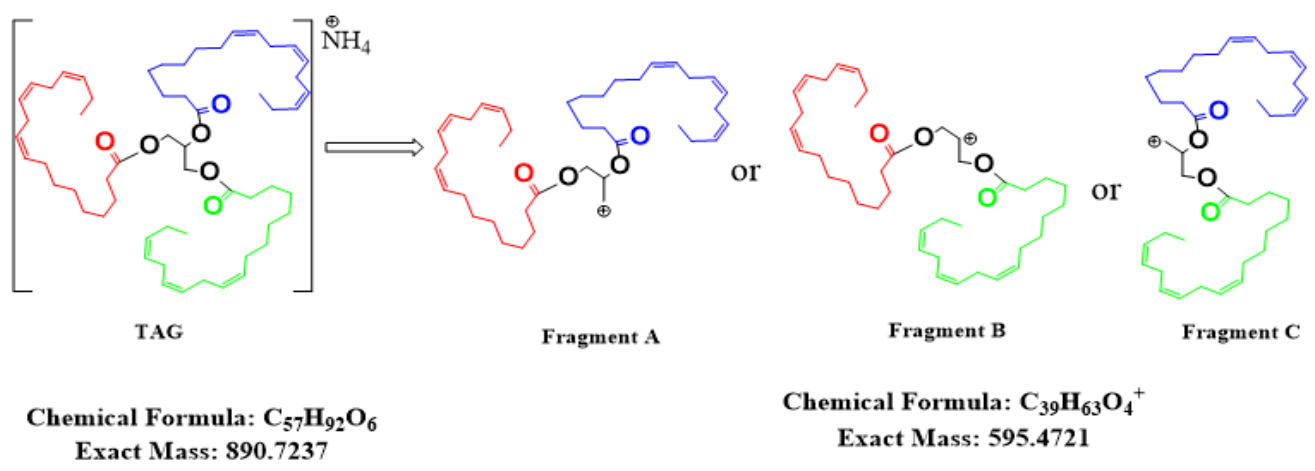

Figure 6. TAG structure and its fragmentation pathway with alternative cleavage on the glycerol structure. 
Furthermore, for the parent ion of 894.7525 with a retention time of $31.87 \mathrm{~min}$, the fragmental ratio of 599.5014 and 595.4655 Daltons is 2:1. Using the same logical evaluation for the parent ion of 896.7682 with the retention time at 32.47 peak min, the TAG structure should have three similar oleic fatty acid chains linked to glycerol, as per hemp seed oil TAG chemical profiles in the literature [31,32]. A summary for those structural assignments is given in Table 2.

Table 2. Fragment summary based on the fragmentation pathway shown in Figure 6. The double bond number is varied from the exact structure in Figure 6.

\begin{tabular}{cccccccc}
\hline$\left[\mathbf{M}+\mathbf{N H}_{4}\right]^{+}$ & Total Double Bond & Red & Blue & Green & Fragment-A & Fragment-B & Fragment-C \\
\hline 890.7216 & 9 & 3 & 3 & 3 & 595 & 595 & 595 \\
\hline 892.7375 & 8 & 2 & 3 & 3 & 597 & 597 & 595 \\
\hline 894.7525 & 7 & 1 & 3 & 3 & 599 & 599 & 595 \\
\hline 896.7682 & 6 & 2 & 2 & 2 & 599 & 599 & 599 \\
\hline
\end{tabular}

Finally, the double bond positions for the peak at $30.32 \mathrm{~min}$ can be further confirmed via its fragmentation patterns. The observation and interpretation in Figures 5 and 6 exclude the chain length variation within three fatty acid chains. However, isomers with double bond position variations are still possible. Common structural isomers such as alpha-linolenic acid (ALA) and gamma-linolenic acid (GLA) fatty acid chains are shown in Figure 7 based on literature reports $[31,35]$.

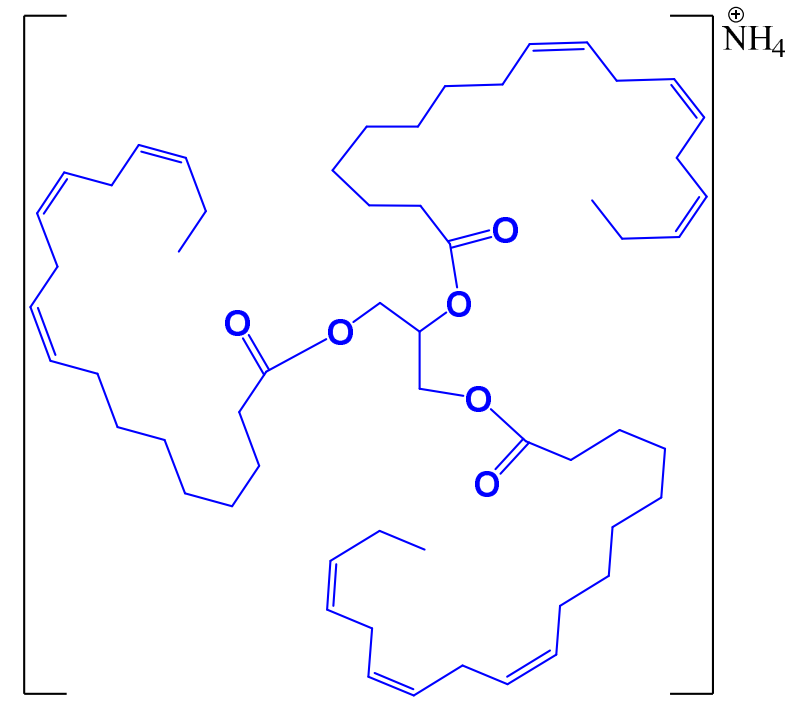

(A)

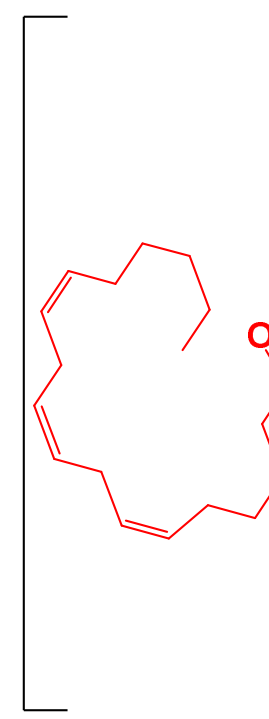

$(\mathbf{B})$

Figure 7. (A) Glycerol, tri- $\omega$-linolenicate $\left[\mathrm{M}+\mathrm{NH}_{4}\right]^{+}$; (B) glycerol, tri- $\gamma$-linolenicate $\left[\mathrm{M}+\mathrm{NH}_{4}\right]^{+}$.

To differentiate these two structures, further fragmentation analyses were executed for the data presented in Figure 8 with an applied NCE level of 30. In Figure 7, the numbers and structures are theoretical fragment weights, and vertical numbers with high resolution mass spectrometric peak are experimental data. The fragments represented by the blue color are from structure 7A. Its theoretical fragments are matched with the experimental data shown in Figure 8. At the same time, the theoretical fragment weight with red color structures shown in Figure 8 are either very weak, such as 97.1004, 123.1160 and 137.1312 in the experimental data, or not observed, such as 111.1174 and 151.1487 from theoretical numbers. If the parent ion concentration is too low, signal ions could be suppressed. The experimental data shown in Figure 8 indicate structure 7A should be dominant in 
hempseed oil. This observation corresponds to the results described in the literature $[31,35]$. The TAG with gamma-linolenic acid composition should be much lower than the TAG with alpha-linolenic acid composition in hemp seed oil. The peak at $30.32 \mathrm{~min}$ is a unique TAG for hemp seed oil. It not only can be representative of hemp seed oil antioxidant benefits but can also avoid interference from skin surface TAG. Therefore, it can be used as the best chemical marker to quantify the hemp seed oil TAG.

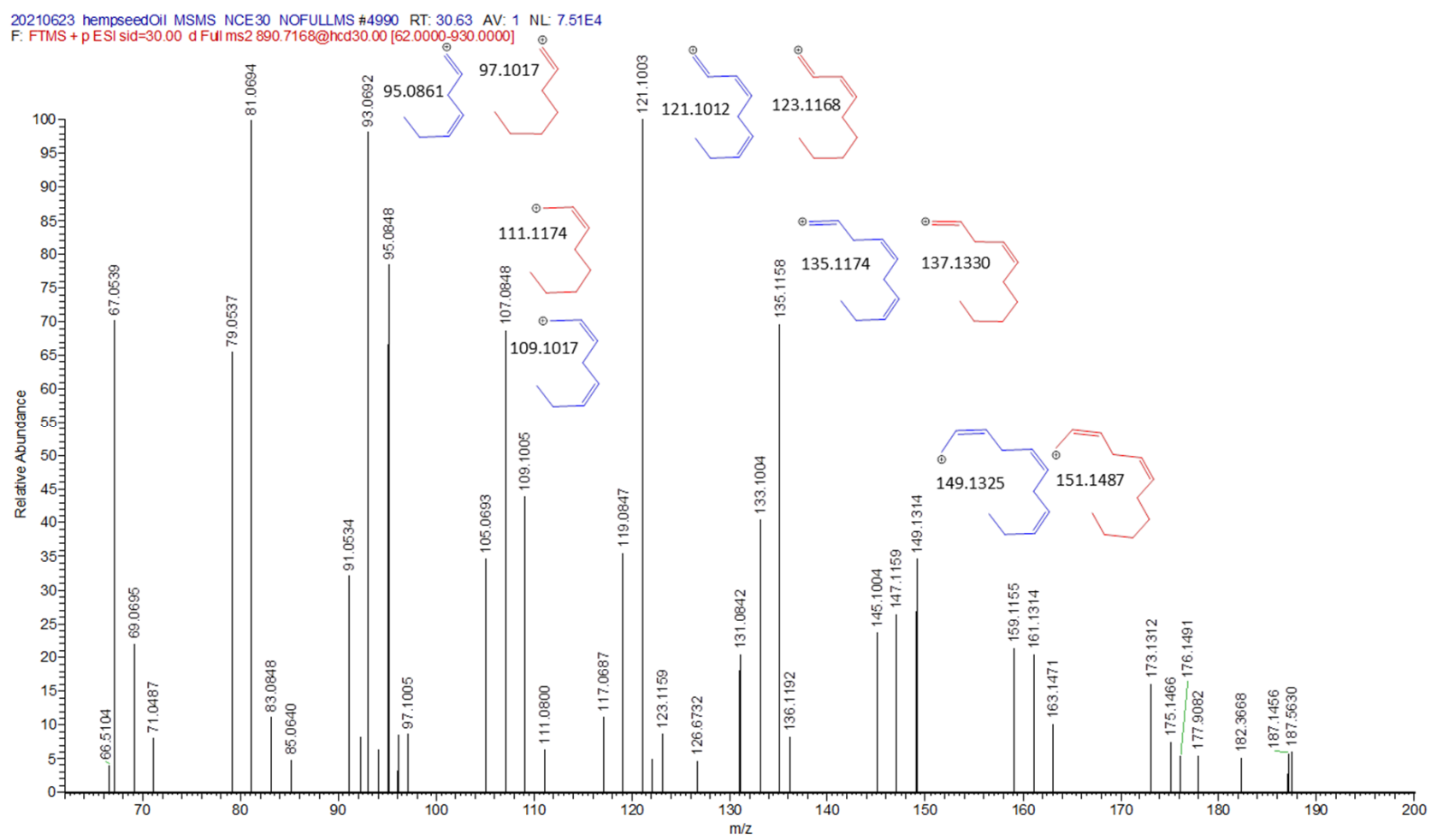

Figure 8. Daughter ions with predicted fragment structures. The horizontal numbers with structure are theoretic fragment weights, and vertical numbers with mass spectra are experimental data.

\subsection{HPLC-HRMS Method Validation}

The method linearity was validated with six batches of standard working solutions. The concentrations, 4.5, 17.8, 71.3 and $285 \mathrm{ppb}$ for CBD and 0.089, 0.356, 1.425 and $5.7 \mathrm{ppm}$ for hemp seed oil, respectively, are shown in Table 1. Correlation coefficients $\left(R^{2}\right)$ were $>0.95$ for all calibration curves. The within-run precision and accuracy were assessed by analyzing six replicated spiked QC samples at medium concentrations of $55 \mathrm{ppb}$ for CBD and $1.1 \mathrm{ppm}$ for hemp seed oil. The within-run accuracy was $-4.53 \%$ for CBD and $2.98 \%$ for hemp seed oil. The within-run precision (RSD) was 3.69\% for CBD and $4.19 \%$ for hemp seed oil. These were all less than $\pm 5.0 \%$, which indicates good accuracy and precision of the method. The method sensitive was defined by the instrumental LOD and LOQ for CBD and hemp seed oil. They were 1.2 and $3.6 \mathrm{ppb}$ for CBD and 0.025 and $0.075 \mathrm{ppm}$ for hemp seed oil.

\section{Conclusions}

By applying normal propanol in the mobile phase with a reverse phase HPLC system, an effective separation methodology was developed. The method can be used to quantify CBD and hemp seed oil simultaneously for skin deposition studies. High resolution mass spectrometry detection combined with data-dependent acquisition provided reliable structural information to support confidence of the quantitation methodology. Glycerol with three alpha linolenic fatty acid chains is well represented as the main ingredients for hemp seed oil activities such as antioxidant and anti-inflammatory activity. This chemical marker was successfully separated from other TAG ingredients either in hemp seed oil 
or pig and human skin surface. More importantly, its structure was well identified and characterized by utilizing tandem mass spectrometry technology, which has not been done previously.

Author Contributions: Conceptualization and methodology design, Z.H.; investigation, software and formal analysis, Y.L. and Y.W.; supervision, L.P. All authors have read and agreed to the published version of the manuscript.

Funding: This research received no external funding.

Institutional Review Board Statement: Not applicable.

Informed Consent Statement: Not applicable.

Conflicts of Interest: The authors declare no conflict of interest.

\section{References}

1. Iffland, K.; Grotenhermen, F. An Update on Safety and Side Effects of Cannabidiol: A Review of Clinical Data and Relevant Animal Studies. Cannabis Cannabinoid Res. 2017, 2, 139-154. [CrossRef]

2. Bergamaschi, M.M.; Queiroz, R.H.C.; Zuardi, A.W.; Crippa, J.A.S. Safety and Side Effects of Cannabidiol, a Cannabis sativa Constituent. Curr. Drug Saf. 2011, 6, 237-249. [CrossRef]

3. Schettino, L.; Prieto, M.; Benedé, J.; Chisvert, A.; Salvador, A. A Rapid and Sensitive Method for the Determination of Cannabidiol in Cosmetic Products by Liquid Chromatography-Tandem Mass Spectrometry. Cosmetics 2021, 8, 30. [CrossRef]

4. Oláh, A.; Tóth, B.I.; Borbíró, I.; Sugawara, K.; Szöllõsi, A.G.; Czifra, G.; Pál, B.; Ambrus, L.; Kloepper, J.; Camera, E.; et al. Cannabidiol exerts sebostatic and antiinflammatory effects on human sebocytes. J. Clin. Investig. 2014, 124, 3713-3724. [CrossRef] [PubMed]

5. Casares, L.; García, V.; Garrido-Rodriguez, M.; Millán, E.; Collado, J.A.; García-Martín, A.; Peñarando, J.; Calzado, M.A.; de la Vega, L.; Muñoz, E. Cannabidiol induces antioxidant pathways in keratinocytes by targeting BACH1. Redox Biol. 2020, 28, 101321. [CrossRef]

6. Jhawar, N.; Schoenberg, E.; Wang, J.V.; Saedi, N. The growing trend of cannabidiol in skincare products. Clin. Dermatol. 2019, 37, 279-281. [CrossRef]

7. Jeong, S.; Kim, M.S.; Lee, S.H.; Park, B.D. Epidermal Endocannabinoid System (EES) and its Cosmetic Application. Cosmetics 2019, 6, 33. [CrossRef]

8. Pundir, S.; Garg, P.; Dviwedi, A.; Ali, A.; Kapoor, V.; Kapoor, D.; Kulshrestha, S.; Lal, U.R.; Negi, P. Ethnomedicinal uses, phytochemistry and dermatological effects of Hippophae rhamnoides L.: A review. J. Ethnopharmacol. 2021, 266, 113434. [CrossRef] [PubMed]

9. Vellido-Perez, J.; Ochando-Pulido, J.; la Fuente, E.B.-D.; Martinez-Ferez, A. Novel emulsions-based technological approaches for the protection of omega-3 polyunsaturated fatty acids against oxidation processes-A comprehensive review. Food Struct. 2021, 27, 100175. [CrossRef]

10. Mayser, P.; Mrowietz, U.; Arenberger, P.; Bartak, P.; Buchvald, J.; Christophers, E.; Jablonska, S.; Salmhofer, W.; Schill, W.-B.; Krämer, H.-J.; et al. $\omega-3$ Fatty acid-based lipid infusion in patients with chronic plaque psoriasis: Results of a double-blind, randomized, placebo-controlled, multicenter trial. J. Am. Acad. Dermatol. 1998, 38, 539-547. [CrossRef]

11. Wang, Y.; Hao, Z.; Pan, L. HRMS Detector for the New HILIC CBD Method Development in Hemp Seed Oil. J. Am. Soc. Mass Spectrom. 2020, 32, 1919-1927. [CrossRef] [PubMed]

12. Picardo, M.; Ottaviani, M.; Camera, E.; Mastrofrancesco, A. Sebaceous gland lipids. Dermato-Endocrinology 2009, 1, 68-71. [CrossRef]

13. Tascini, A.S.; Noro, M.G.; Seddon, J.M.; Chen, R.; Bresme, F. Mechanisms of lipid extraction from skin lipid bilayers by sebum triglycerides. Phys. Chem. Chem. Phys. 2019, 21, 1471-1477. [CrossRef] [PubMed]

14. Radner, F.P.; Grond, S.; Lass, A.; Zechner, R. Fat in the skin: Triacylglycerol metabolism in keratinocytes and its role in the development of neutral lipid storage disease. Dermato-Endocrinology 2011, 3, 77-83. [CrossRef] [PubMed]

15. Rodríguez-Alcalá, L.; Fontecha, J. Major lipid classes separation of buttermilk, and cows, goats and ewes milk by high performance liquid chromatography with an evaporative light scattering detector focused on the phospholipid fraction. J. Chromatogr. A 2010, 1217, 3063-3066. [CrossRef]

16. Indelicato, S.; Bongiorno, D.; Pitonzo, R.; Di Stefano, V.; Calabrese, V.; Indelicato, S.; Avellone, G. Triacylglycerols in edible oils: Determination, characterization, quantitation, chemometric approach and evaluation of adulterations. J. Chromatogr. A 2017, 1515, 1-16. [CrossRef]

17. Yang, Q.; Shi, X.; Gu, Q.; Zhao, S.; Shan, Y.; Xu, G. On-line two dimensional liquid chromatography/mass spectrometry for the analysis of triacylglycerides in peanut oil and mouse tissue. J. Chromatogr. B 2012, 895-896, 48-55. [CrossRef] [PubMed]

18. Han, X.; Ye, H. Overview of Lipidomic Analysis of Triglyceride Molecular Species in Biological Lipid Extracts. J. Agric. Food Chem. 2021, 69, 8895-8909. [CrossRef] [PubMed] 
19. Ruiz-Samblás, C.; González-Casado, A.; Rodríguez, L.C. Triacylglycerols Determination by High-temperature Gas Chromatography in the Analysis of Vegetable Oils and Foods: A Review of the Past 10 Years. Crit. Rev. Food Sci. Nutr. 2013, 55, 1618-1631. [CrossRef]

20. Castro-Gómez, M.P.; Holgado, F.; Rodríguez-Alcalá, L.M.; Montero, O.; Fontecha, J. Comprehensive Study of the Lipid Classes of Krill Oil by Fractionation and Identification of Triacylglycerols, Diacylglycerols, and Phospholipid Molecular Species by Using UPLC/QToF-MS. Food Anal. Methods 2015, 8, 2568-2580. [CrossRef]

21. Buchgraber, M.; Ulberth, F.; Emons, H.; Anklam, E. Triacylglycerol profiling by using chromatographic techniques. Eur. J. Lipid Sci. Technol. 2004, 106, 621-648. [CrossRef]

22. Balgoma, D.; Guitton, Y.; Evans, J.J.; Le Bizec, B.; Dervilly-Pinel, G.; Meynier, A. Modeling the fragmentation patterns of triacylglycerides in mass spectrometry allows the quantification of the regioisomers with a minimal number of standards. Anal. Chim. Acta 2019, 1057, 60-69. [CrossRef]

23. Renaud, J.B.; Overton, S.; Mayer, P.M. Energy and entropy at play in competitive dissociations: The case of uneven positional dissociation of ionized triacylglycerides. Int. J. Mass Spectrom. 2013, 352, 77-86. [CrossRef]

24. Franklin, E.T.; Xia, Y. Structural elucidation of triacylglycerol using online acetone Paternò-Büchi reaction coupled with reversedphase liquid chromatography mass spectrometry. Analyst 2020, 145, 6532-6540. [CrossRef] [PubMed]

25. Williams, P.E.; Klein, D.R.; Greer, S.M.; Brodbelt, J.S. Pinpointing Double Bond and sn-Positions in Glycerophospholipids via Hybrid 193 nm Ultraviolet Photodissociation (UVPD) Mass Spectrometry. J. Am. Chem. Soc. 2017, 139, 15681-15690. [CrossRef]

26. Kodali, S.; Shukla, V.K.S. CBD Oil for Healthier-Looking Skin; International Cosmetics Science Center A/S: Aarhus, Denmark, 2019; Volume 48 .

27. Liao, F.-Y.; Lin, Y.-C.; Chen, Y.-L.; Feng, C.-H. Determination of tranexamic acid in various matrices using microwave-assisted derivatization followed by dispersive liquid-liquid microextraction. J. Chromatogr. A 2015, 1377, 35-45. [CrossRef]

28. Paulson, D.S.; Topp, R.; Boykin, R.E.; Schultz, G.; Yang, Q. Efficacy and safety of a novel skin cleansing formulation versus chlorhexidine gluconate. Am. J. Infect. Control 2018, 46, 1262-1265. [CrossRef]

29. Wertz, P.W.; Downing, D.T. Covalently bound w-hydroxyacylsphingosine in the stratum corneum. Biochim. Biophys. Acta (BBA) Lipids Lipid Metab. 1987, 917, 108-111. [CrossRef]

30. Pinna, K.; Whitney, E.; Rady Rolfes, S. Understanding Nutrition, 11th ed.; Brooks Cole Publishing Company: Pacific Grove, CA, USA, 2015; pp. 144-147.

31. Alonso-Esteban, J.I.; Fernández, M.J.G.; Fabrikov, D.; Torija-Isasa, E.; Sánchez-Mata, M.D.C.; Guil-Guerrero, J.L. Hemp (Cannabis sativa L.) Varieties: Fatty Acid Profiles and Upgrading of $\gamma$-Linolenic Acid-Containing Hemp Seed Oils. Eur. J. Lipid Sci. Technol. 2020, 122, 1900445. [CrossRef]

32. Abdollahi, M.; Sefidkon, F.; Calagari, M.; Mousavi, A.; Mahomoodally, M.F. A comparative study of seed yield and oil composition of four cultivars of Hemp (Cannabis sativa L.) grown from three regions in northern Iran. Ind. Crops Prod. 2020, $152,112397$. [CrossRef]

33. Marzocchi, S.; Caboni, M. Effect of harvesting time on hemp (Cannabis sativa L.) seed oil lipid composition. Ital. J. Food Sci. 2020, $32,1018-1029$.

34. Tringaniello, C.; Cossignani, L.; Blasi, F. Characterization of the Triacylglycerol Fraction of Italian and Extra-European Hemp Seed Oil. Foods 2021, 10, 916. [CrossRef] [PubMed]

35. Ianni, F.; Blasi, F.; Giusepponi, D.; Coletti, A.; Galli, F.; Chankvetadze, B.; Galarini, R.; Sardella, R. Liquid chromatography separation of $\alpha$ - and $\gamma$-linolenic acid positional isomers with a stationary phase based on covalently immobilized cellulose tris(3,5-dichlorophenylcarbamate). J. Chromatogr. A 2020, 1609, 460461. [CrossRef] [PubMed] 